\title{
A Case Report of Massive Unilateral Pleural Effusion in a Patient with Systemic Lupus Erythematosus Responded to Rituximab
}

\author{
Fatima Magzoub Mohamed Khatieb ${ }^{1,2}$, Sara Hamza Abushama ${ }^{1}$, \\ Mohammed Elmujtba Adam Essa Adam ${ }^{3,4,5^{*}} \mathbb{0}$, Shaima N. Elgenaid 6 , \\ Abdelkareem A. Ahmed ${ }^{3,7,8,9}$, Malaz Faiz Abdelrahman Elkhadir ${ }^{10}$, Ziryab Imad Taha ${ }^{3,11}$, \\ Ayman Sati Sati Mohamed ${ }^{3,4}$, Sadia Kamal Albadawi Mohamed ${ }^{12}$ and Elnour Mohammed Elagib ${ }^{1,13}$ \\ ${ }^{1}$ Department of Rheumatology, Sudan Medical Specialization Board, Khartoum, Sudan; ${ }^{2}$ Department of Medicine, Almughtaribeen Uni- \\ versity, Khartoum, Sudan; ${ }^{3}$ Departments of Clinical Medicine, Medical and Cancer Research Institute (MCRI), Nyala, Sudan; ${ }^{4}$ Faculty \\ of Medicine, Al Fashir University, Al Fashir, Sudan; ${ }^{5}$ Research Department, Sudanese Medical Research Association, Khartoum, Sudan; \\ ${ }^{6}$ Faculty of Medicine, University of Khartoum, Khartoum, Sudan; ${ }^{7}$ Department of Physiology and Biochemistry, Faculty of Veterinary Sci- \\ ence, University of Nyala, Nyala, Sudan; ${ }^{8}$ Biomedical Research Institute, Darfur University College, Nyala, Sudan; ${ }^{9}$ Institute of Molecular \\ Biology, University of Nyala, Nyala, Sudan; ${ }^{10}$ Faculty of Medicine, International University of Africa, Khartoum, Sudan; ${ }^{11}$ Department of \\ Internal Medicine, Faculty of Medicine, University of Bahri, Khartoum, Sudan; ${ }^{12}$ Department of Medicine, Sudan Medical Specializations \\ Board, Khartoum, Sudan; ${ }^{13}$ Department of Medicine and Rheumatology, Omdurman Military Hospital, Sudan
}

\begin{abstract}
Systemic lupus erythematosus (SLE) is a systemic disease which affects mainly young females and can cause lifethreatening conditions. Pleural effusion can occur in SLE patients and usually tends to be mild and bilateral. This report aims to highlight the clinical presentation and medical management of massive unilateral pleural effusions in SLE patients. Here we report a 35-year-old female diagnosed with SLE for six years. She presented with shortness of breath, severe pleuritic chest pain, and fatigue. Her clinical examination showed signs of massive pleural effusion on the right side which was confirmed later by a chest $x$-ray and computer tomography of the chest. An echocardiography and abdominal ultrasound indicated no pericardial effusion and no ascites. A pleural fluid analysis showed exudative fluid. Sputum culture and polymerase chain reaction on blood sample for Mycobacterium tuberculosis were negative. She was also edematous and pale but not cyanotic or jaundiced. The treatment included blood transfusions, antibiotics, rituximab, azathioprine, and hydroxychloroquine. The pleural effusion responded well to rituximab, and she was discharged after two months in good condition.
\end{abstract}

Introduction

Systemic lupus erythematosus (SLE) is a chronic autoimmune

Keywords: Massive; Unilateral; Pleural effusion; Systemic lupus erythematosus Rituximab.

Abbreviations: ANA, antinuclear antibodies; CRP, C-reactive protein; CT, computed tomography; ESR, erythrocytes sedimentation rate; ECHO, Echocardiography; HCQ, hydroxychloroquine; LDH, lactate dehydrogenase; MMF, mycophenolate mofetil; NSAIDs, non-steroidal anti-inflammatory drugs; PCR, polymerase chain reaction; SLE, systemic lupus erythematosus.

Received: September 26, 2020; Revised: October 27, 2020; Accepted: November 12 , 2020

*Correspondence to: Mohammed Elmujtba Adam Essa Adam, Department of Clinical Medicine, Medical and Cancer Research Institute, Nyala, Sudan. ORCID: https://orcid.org/0000-0002-1050-2771. Tel: 00249907009389,E-mail: Awadali818@ yahoo.com

How to cite this article: Khatieb FMM, Abushama SH, Essa Adam MEA, Elgenaid SN, Ahmed AA, Elkhadir MFA, et al. A Case Report of Massive Unilateral Pleura Effusion in a Patient with Systemic Lupus Erythematosus Responded to Rituximab. Exploratory Research and Hypothesis in Medicine 2020;000(000):000-000. doi: 10.14218/ERHM.2020.00057. disease of unknown origin affecting predominantly females at a young age with no predilection for specific organs. ${ }^{1,2}$ The overall prevalence of SLE is $0.2-1.5 / 1,000 .{ }^{3}$ However, the epidemiology remains undetected in many developing countries. ${ }^{4}$ In Sudan, many cases have been misdiagnosed due to low recognition of the disease at its early stages.

SLE may manifest with various features, such as fatigue, fever, arthritis, skin rash, and joint pain. It can cause severe life-threatening conditions by damaging the organs and tissues. ${ }^{2}$ Pleural effusion occurs in $16-50 \%$ of SLE patients and usually tends to be bilateral and minimal. ${ }^{5}$ However, massive unilateral pleural effusion in SLE is rarely reported. This case report aims to draw attention to the presentation and medical management of massive unilateral pleural effusion in SLE.

\section{Case report}

The patient is a 35 -year-old housewife who is married with no children. Initially, she presented on March 2011 with a sudden onset 
history of joint pain, fatigue, a facial rash, hair loss, and oral and genital ulcers. Two months later, she developed loss of memory, confusion, convulsions, epistaxis, and rectal bleeding. Investigations showed low platelets count and a positive antinuclear antibodies (ANA) profile, anti dsDNA, anti-histones, and anti-sm antibodies (Table 1). Therefore she was diagnosed with SLE based on the clinical as well as the laboratory evidence. Consequently, she received pulse corticosteroids (1,000 $\mathrm{mg} /$ once per day for 3 days), azathioprine (50 mg every 12 hours), hydroxychloroquine (HCQ) (200 mg twice daily), and oral corticosteroids (10 mg once daily).

Her condition improved except for the low platelets count, for which she received mycophenolate mofetil (MMF), $500 \mathrm{mg}$ twice daily, for 6 months. During this period, the azathioprine was discontinued. After six months of using MMF she continued on the previous drug regimen (HCQ) and azathioprine). Her condition was well controlled with her medications. In 2016, azathioprine and HCQ were discontinued and methotrexate was started in Thailand as she reported a history of joint pain at that time. After that, she developed bullous skin rashes over her face (Fig. 1), trunk, and arms. It was painful, itchy, and scarring. Therefore, methotrexate was stopped, and MMF was restarted. She experienced a good response; however, a few months later, she developed lower limb edema and elevated blood pressure. Investigations revealed high 24-hour urinary proteins, blood urea, and creatinine levels. Subsequently, belimumab (1,200 mg inf/month) was prescribed. After receiving two dosages of belimumab, the patient developed generalized body swelling and a new urticarial and purpuric rash in addition to the previous skin rash (Fig. 2). As a result, she was admitted to the hospital, the belimumab was stopped and she received diuretics, anti-hypertensive, MMF, and HCQ. Her condition then improved well. Six months later, the patient presented with shortness of breath, pleuritic chest pain, and fatigue. On examination, she was edematous and pale but not cyanotic or jaundiced. Her vital signs were normal, and a chest examination revealed signs of massive unilateral pleural effusion on the right side. A skin examination showed areas of hypo and hyperpigmentation with scarring and alopecia. Investigations showed elevated erythrocytes sedimentation rate (ESR), blood urea, and creatinine levels in addition to low complement levels (Table 1). A chest X-ray detected a massive right-sided pleural effusion, and a chest computed tomography (CT) showed right hydropneumothorax with shifted mediastinum (Fig. 3). An echocardiograph study was normal, and an abdominal ultrasound was negative for ascites with normal-sized kidneys and normal echogenicity. A pleural fluid analysis showed an accumulation of exudative fluid. And, since tuberculosis is common in Sudan, sputum culture and polymerase chain reaction (PCR) on blood sample for Mycobacterium tuberculosis were requested and both were negative. Also, the pleural fluid culture and sensitivity were negative. A pleural biopsy showed active chronic pleurisy (Table 2). The blood culture was done and was negative. The immune modulator medications were stopped, and she received antibiotics and a blood transfusion. Repeated pleural fluid aspirations of 2-2.5 L each time were performed. She received three haemodialysis sessions, and a chest tube was inserted which drained more than $3 \mathrm{~L}$. Therefore, a pulmonologist was consulted for pleurodesis. Later, her condition improved and a chest tube was removed. She became asymptomatic and was advised to start rituximab. Her renal function normalized, and she was discharged in good condition and symptom free (Fig. 1c). HCQ (200 mg twice daily), azathioprine (100 $\mathrm{mg}$ in the morning and $50 \mathrm{mg}$ in the evening), and prednisolone were prescribed. On the $9^{\text {th }}$ of January, she received the first rituximab dose. The patient is now showing a very good response with more than one year without flare ups.

\section{Discussion}

About $4-5 \%$ of patients with SLE can present with pulmonary involvement as an initial manifestation, while $50 \%$ of the patients can present with pulmonary manifestation as late complications. ${ }^{6}$ SLE can cause pleural disease, acute lupus pneumonitis, diffuse alveolar haemorrhage, chronic interstitial disease, pulmonary hypertension, pulmonary embolism, and other conditions; however, pleuritis is the most common pulmonary manifestation. ${ }^{7}$ Around half of the patients with pleuritic involvement had pleural effusion, and $93 \%$ had evidence of pleuritis at autopsy. ${ }^{7}$ In SLE, pleural effusion can be primary, such as that caused by lupus pleuritis, or secondary to other causes, such as infection, heart failure, pulmonary embolism, or renal failure.?

SLE usually presents with pleuretic chest pain, dyspnea, fever, and cough. ${ }^{7}$ Our patient presented with shortness of breath and chest pain. Her examination showed features of right-sided massive pleural effusion confirmed by a chest x-ray and CT scan. Pleural effusion in SLE tends to be bilateral and usually small-tomoderate in size, but, occasionally, it can be a massive effusion, and pleural fluid analysis is essential for diagnosis. ${ }^{8}$ It is typically exudative effusion with a high leukocyte count with prominent neutrophils.

Exudative pleural effusion occurs either due to entering of protein and cells through a highly permeable mesothelial membrane or due to impaired removal of proteins and cells. ${ }^{9}$ A biochemical analysis usually shows high proteins (pleural fluid/serum ratio greater than 0.5 ), elevated lactate dehydrogenase (LDH) (pleural fluid/serum ratio greater than 0.6 ), and normal or low glucose levels. ${ }^{7}$ In addition, a high ANA titer of at least 1:160 in pleural fluid and elevated serum C-reactive protein (CRP) levels can be indicative of lupus pleuritis. ${ }^{10,11}$ This patient underwent thoracocentesis, and the biochemical analysis of pleural fluid revealed normal glucose, high protein levels, and a raised neutrophil count, which was consistent with exudative fluid. Her CT scan showed a massive right-sided pleural effusion with mediastinum shift. Regarding the hydropneumothorax, it more likely developed as a complication of a chest tube insertion.

Heart failure, pulmonary tuberculosis, and lupus nephritis can be possible causes of pleural effusion in SLE patients. In a study done by Palavutitotai N et al. ${ }^{12}$ among 119 SLE patients, $37 \%$ presented with pleuritis as a first presentation and was accompanied by pericarditis in $16 \%$. The most common cause of pleural effusion was found to be lupus pleuritis (52\%), and other causes were tuberculous pleuritis $(9 \%)$, parapneumonic effusion $(7 \%)$, and transudate $(15 \%))^{12}$ Our patient had negative sputum and PCR tests for Mycobacterium tuberculosis and a normal Echocardiography (ECHO) and abdominal ultrasound. Blood and pleural fluid cultures and sensitivity were negative. Patients with an active flare of SLE usually have normal CRP and high ESR. A high level of CRP is more indicative of infection in SLE patients rather than an active disease. ${ }^{13}$ Therefore, investigations of this patient excluded tuberculosis, heart failure, and infection as underlying causes of the pleural effusion.

The patient had recurrent flare ups of the disease as indicated by her symptoms of joint pain, skin rash, hair loss, and ulcers. Moreover, investigations during the last visit showed high urea and creatinine levels and low complement levels consistent with lupus nephritis, but the patient refused a renal biopsy. Active SLE was documented in $81 \%$ of patients with pleural effusion. ${ }^{12}$ In a case reported by Castaneda et al., ${ }^{14}$ an African American female with a history of SLE presented with massive pleural effusion and concomitant class III lupus nephritis confirmed with a biopsy. Her 
Table 1. General test results of the patient

\begin{tabular}{|c|c|c|}
\hline Time & Investigation & Result \\
\hline \multirow[t]{7}{*}{2011} & antinuclear antibodies & $1 / 2,000$ \\
\hline & dsDNA & Positive $(++)$ \\
\hline & Nucleosomes & Positive $(+++)$ \\
\hline & Histones & Positive (+++) \\
\hline & Sm antibody & Positive $(+++)$ \\
\hline & anti-Sjögren's-syndrome-related antigen A autoantibodies & Positive $(++)$ \\
\hline & Serum anti-mitochondrial antibody type 2 & Positive (+) \\
\hline \multirow[t]{10}{*}{2016} & Complete Blood Count & Normal \\
\hline & C-reactive Protein & 5 \\
\hline & Erythrocytes sedimentation rate & 30 \\
\hline & Total protein & 5.3 \\
\hline & Serum albumin & 3 \\
\hline & aspartate aminotransferase & 11 \\
\hline & alanine aminotransferase & 33 \\
\hline & $24 \mathrm{~h}$ urinary protein & $2,968.2 \mathrm{mg} / 24 \mathrm{~h}$ \\
\hline & Urea & $55 \mathrm{mg} / \mathrm{dl}$ \\
\hline & S. Creatinine & $1.6 \mathrm{mg} / \mathrm{dl}$ \\
\hline \multirow[t]{19}{*}{2018} & White Blood Cells & 5.3 \\
\hline & Hemoglobin & 9.1 \\
\hline & Platelet & 210 \\
\hline & Erythrocytes Sedimentation Rate & 70 \\
\hline & C-reactive protein & 1.8 \\
\hline & Complement 3 and Complement 4 levels & Low \\
\hline & Immunoglobulin levels & Normal \\
\hline & Bleeding profile & Normal \\
\hline & Urea & 67 raising to 173 \\
\hline & Creatinine & 1.74 raising to 5.6 \\
\hline & Potassium & 4.3 raising to 7 \\
\hline & Uric acid & 8.1 \\
\hline & Serum albumin & 3.1 \\
\hline & Globulin levels & 4.2 \\
\hline & Urine pus cells & $10-12$ \\
\hline & Urine Red Blood Cells & $8-10$ \\
\hline & Urine protein & + \\
\hline & Urine cast & No \\
\hline & Blood culture & Negative \\
\hline \multirow[t]{5}{*}{ Before discharge } & Complete Blood Count & Normal \\
\hline & Erythrocytes Sedimentation Rate & 37 \\
\hline & C-reactive Protein & 0.5 \\
\hline & Urea & 1.3 \\
\hline & Serum Creatinine & 42 \\
\hline
\end{tabular}



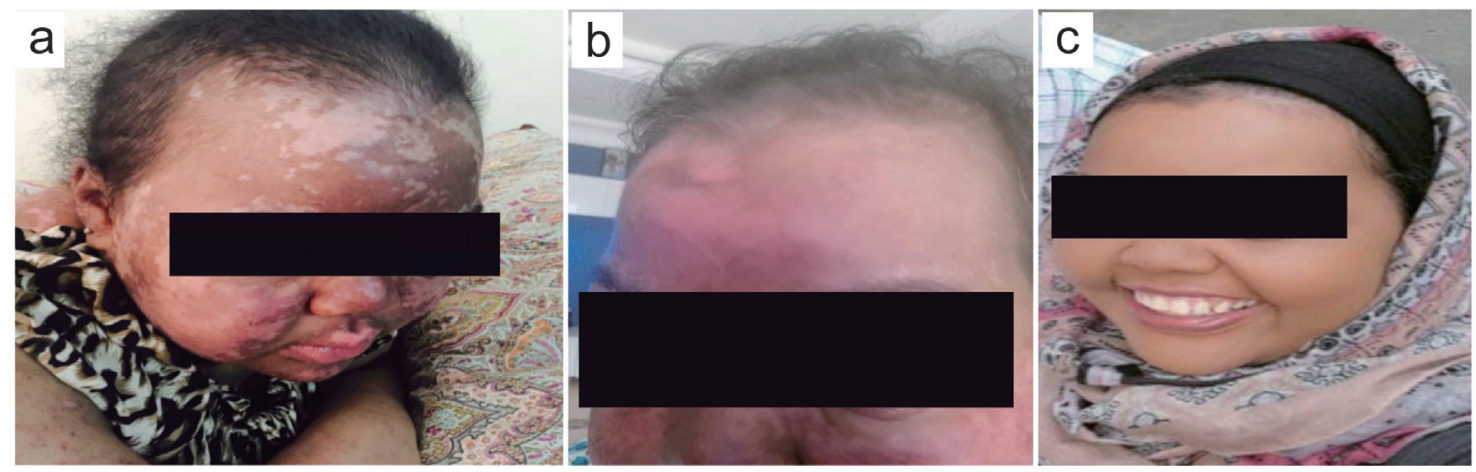

Fig. 1. Photos of the patient during the presentation and after discharge. (a) Facial skin rash, (b) edema, and a (c) skin rash and edema subsided after treatment with rituximab.
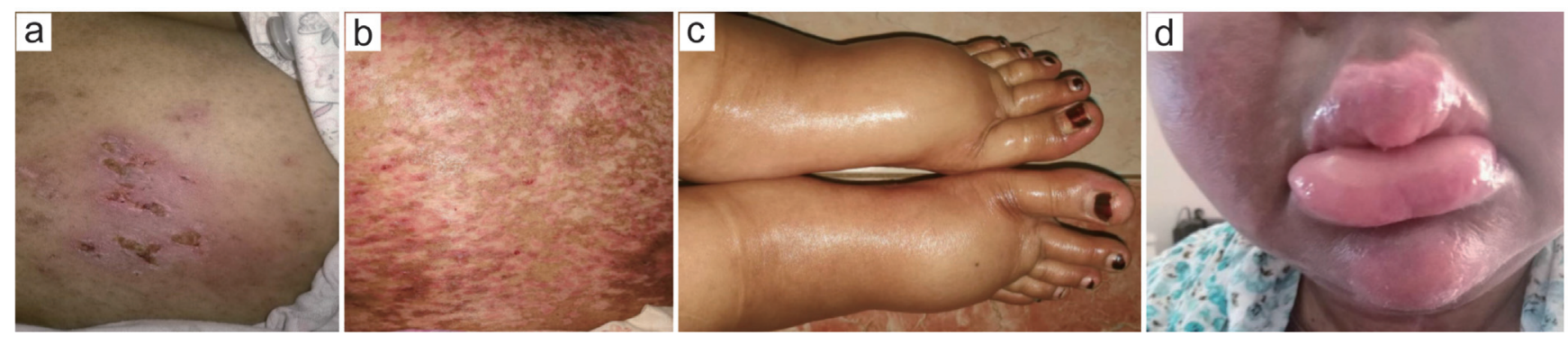

Fig. 2. The skin rash and generalized body edema of the patient. (a) Bullous skin rash with scarring; (b) skin rash on the trunk; (c) lower limb edema; and (d) facial edema.

condition improved, and both lupus nephritis and effusion were resolved with a four-week use of prednisone and MMF. ${ }^{14}$ This presentation is unique as it is a unilateral effusion unlike the usual bilateral pleural effusion in SLE.

Typical therapy for SLE consists of non-steroidal anti-inflammatory drugs (NSAIDs), HCQ, glucocorticoids, and immunosuppressive medications, but the drugs should be prescribed according to clinical manifestations. NSAID is recommended for fever, serositis, and musculoskeletal symptoms, and HCQ is helpful for skin involvement as well. Patients with severe disease and organ involvement (especially renal and central nervous system involvement) will benefit from a high dose of steroids and immunosuppressive medications, such as methotrexate, cyclophosphamide, azathioprine, mycophenolate or rituximab, especially if there is no response to glucocorticoids. MMF was introduced to the patient but stopped after six months due to patient concern about her fertility and the ability to conceive in the near future, as many reports indicated that immunosuppressive drugs in young patients may have deleterious effects on pregnancy, fertility, and teratogenicity to an unborn child. ${ }^{15}$ Recently, belimumab, approved to be the treatment of adult SLE patients with active, autoantibody-positive SLE, who are failed to respond to standard therapy. ${ }^{10,16}$ Some clinical reports indicate that belimumb is usually well-tolerated, but may carry a risk of adverse events, such as infections, hypersensitivity, and infusion reactions, ${ }^{17}$ and, hence, the administration of belimumb was stopped in our patient after the second dose when she reported an allergic reaction. For massive pleural effusion, many cases responded well to hydroxychloroquine, methylprednisolone, azathioprine, and cyclophosphamide..$^{8,10}$ But refractory cases to the previous treatment were documented, and, in such cases, intrave-
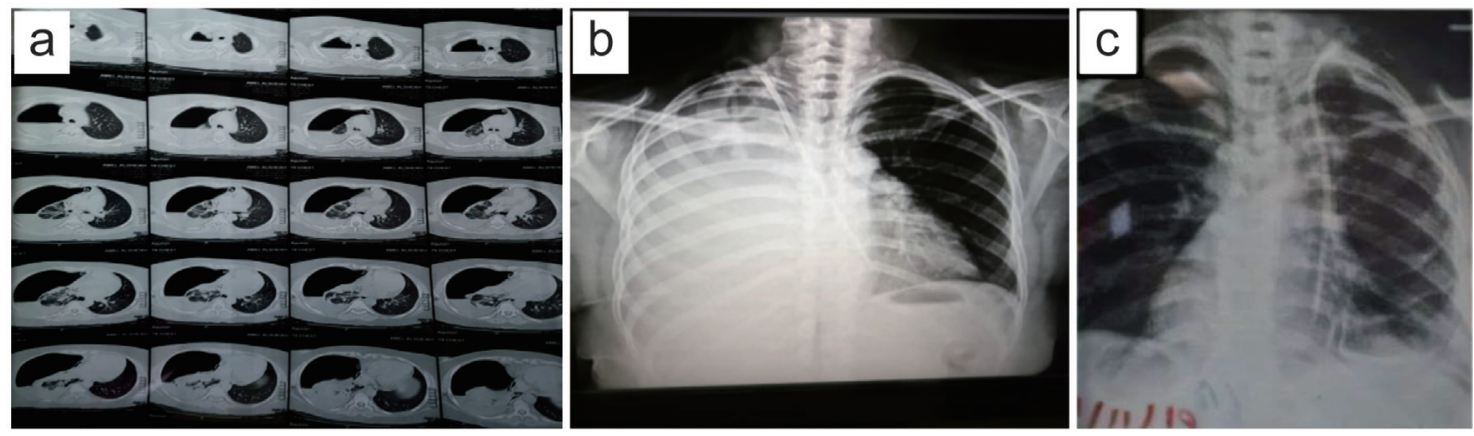

Fig. 3. Imaging examination of the patient. (a) Right side hydro pneumothorax with a mediastinal shift in the CT scan; (b) right side pleural effusion visible in the chest $\mathrm{X}$ ray; and (c) a chest tube on the right side with a decreased amount of fluid. 
Table 2. pleural fluid analysis

\begin{tabular}{lll}
\hline Investigation & Result & Normal Value \\
\hline Protein & $4.2 \mathrm{~g} / \mathrm{dl}$ & Less than $3 \mathrm{~g} / \mathrm{dl}$ \\
Glucose & $101 \mathrm{mg} / \mathrm{dl}$ & $80-180 \mathrm{mg} / \mathrm{dl}$ \\
WBCs & $200(60 \%$ neutrophil-40\% lymphocytes) & Less than 1,000 per cubic millimeter \\
LDH & $99 \mathrm{U} / \mathrm{L}$ & less than $200 \mathrm{U} / \mathrm{L}$ \\
Culture and sensitivity & Negative & \\
Pleural biopsy & Active chronic pleurisy & \\
\hline
\end{tabular}

nous immunoglobulin was considered, and some cases responded well to talc pleurodesis and surgical pleurectomy. ${ }^{5,18}$ In this case, the patient responded to rituximab with clinical and radiological improvement. Few similar cases were documented, as in a case reported by Ocampo V et al., ${ }^{19}$ in which a 22 -year-old male with SLE developed recurrent pericardial and massive unilateral pleural effusion and responded well to tocilizumab. ${ }^{19}$

In summary, SLE and connective tissue disorders are mainly responsible for pleural effusion development, especially among younger females. The usual presentation is bilateral effusion that responds well to glucocorticoids. However, here we presented a case of SLE with unusual massive unilateral pleural effusion and generalized edema which responded well to rituximab.

\section{Acknowledgments}

The authors wish to acknowledge Medical and Cancer research Institute (MCRI) for their support to conduct this research.

\section{Ethical statement}

The authors have obtained the written consent from the patient to publish this case report.

\section{Funding}

This study was funded by Medical and Cancer Research Institute (MCRI), Sudan.

\section{Conflict of interest}

The authors declare that they have no competing interests.

\section{Author contributions}

Manuscript writing and critical revision (MEAEA, SNE, AAA); diagnose and treat the case (FMMK, SHA, EME); data collections and technical editing (ZIT, ASSM, MFAE, SKAM).

\section{References}

[1] Elagib E, Eltahir N, Essa Adam M, Yousif Haron M, Mahmoud Z, Mohamed Yousif H. Catastrophic antiphospholipid syndrome in combi- nation with SLE treated by Rituximab: a case report and literature review. Lupus: Open Access 2019;4(1):137

[2] Askanase A, Shum K, Mitnick H. Systemic lupus erythematosus: an overview. Soc Work Health Care 2012;51(7):576-586. doi:10.1080/0 0981389.2012.683369.

[3] Pons-Estel GJ, Alarcon GS, Scofield L, Reinlib L, Cooper GS. Understanding the epidemiology and progression of systemic lupus erythematosus. Semin Arthritis Rheum 2010;39(4):257-268. doi:10.1016/j. semarthrit.2008.10.007.

[4] Tiffin N, Hodkinson B, Okpechi I. Lupus in Africa: can we dispe the myths and face the challenges? Lupus 2014;23(1):102-111. doi:10.1177/0961203313509296.

[5] Breuer GS, Deeb M, Fisher D, Nesher G. Therapeutic options for refractory massive pleural effusion in systemic lupus erythematosus: a case study and review of the literature. Semin Arthritis Rheum 2005;34(5):744-749. doi:10.1016/j.semarthrit.2004.11.003.

[6] Aguilera-Pickens G, Abud-Mendoza C. Pulmonary manifestations in systemic lupus erythematosus: pleural involvement, acute pneumonitis, chronic interstitial lung disease and diffuse alveolar hemorrhage. Reumatol Clin 2018;14(5):294-300. doi:10.1016/j.reuma.2018.03.012.

[7] Crestani B. The respiratory system in connective tissue disorders. Allergy 2005;60(6):715-734. doi:10.1111/j.1398-9995.2005.00761.x.

[8] McGrath EE, Anderson PB. Diagnosis of pleural effusion: a systematic approach. Am J Crit Care 2011;20(2):119-127; quiz 128. doi:10.4037/ ajcc2011685.

[9] Zocchi L. Physiology and pathophysiology of pleural fluid turnover. Eur Respir J 2002;20(6):1545-1558. doi:10.1183/09031936.02.00062102.

[10] Choi BY, Yoon MJ, Shin K, Lee YJ, Song YW. Characteristics of pleu ral effusions in systemic lupus erythematosus: differential diagnosis of lupus pleuritis. Lupus 2015;24(3):321-326. doi:10.1177/ 0961203314555171.

[11] Callejas Rubio JL, Rios Fernandez R, Moreno Higueras M, Ortego Centeno N. Usefulness of assessment of antinuclear antibodies in pleural effusion for diagnosis of lupus pleuritis (in Spanish ). Med Clin (Barc) 2016;146(7):e37. doi:10.1016/j.medcli.2015.10.034.

[12] Palavutitotai N, Buppajarntham T, Katchamart W. Etiologies and outcomes of pleural effusions in patients with systemic lupus erythematosus. J Clin Rheumatol 2014;20(8):418-421. doi:10.1097/ RHU.0000000000000179.

[13] Wang J, Niu R, Jiang L, Wang $Y$, Shao X, Wu M, et al. The diagnostic values of $C$-reactive protein and procalcitonin in identifying systemic lupus erythematosus infection and disease activity. Medicine 2019;98(33):e16798. doi:10.1097/MD.0000000000016798.

[14] Castaneda JL, Freire AX. Massive Pleural Effusion and Concomitant Lupus Nephritis Flare: The Value of Lupus Erythematous (le) Cells in the Pleural Fluid. Am J Respir Crit Care Med 2015;191:A2949. Available from: https://www.atsjournals.org/doi/abs/10.1164/ajrccm-conference.2015.191.1_MeetingAbstracts.A2949 Accessed November 25, 2020.

[15] Leroy C, Rigot JM, Leroy M, Decanter C, Le Mapihan K, Parent AS, et al. Immunosuppressive drugs and fertility. Orphanet J Rare Dis 2015;10:136. doi:10.1186/s13023-015-0332-8.

[16] Hui-Yuen JS, Reddy A, Taylor J, Li X, Eichenfield AH, Bermudez LM, et al. Safety and Efficacy of Belimumab to Treat Systemic Lupus Erythematosus in Academic Clinical Practices. J Rheumatol 2015;42(12):2288-2295. doi:10.3899/jrheum.150470. 
Explor Res Hypothesis Med

[17] Ginzler EM, Wallace DJ, Merrill JT, Furie RA, Stohl W, Chatham WW, et al. Disease control and safety of belimumab plus standard therapy over 7 years in patients with systemic lupus erythematosus. J Rheumatol 2014;41(2):300-309. doi:10.3899/jrheum.121368.

[18] Song P, Zhang J, Shang C, Zhang L. Refractory chylous pleural effusion with systemic lupus erythematosus: Surgical treatment when
Khatieb FMM. et al: Massive unilateral pleural effusion in SLE patient steroid/immunosuppressant resistant. Z Rheumatol 2019;78(8):797802. doi:10.1007/s00393-018-0545-z.

[19] Ocampo V, Haaland D, Legault K, Mittoo S, Aitken E. Successful treatment of recurrent pleural and pericardial effusions with tocilizumab in a patient with systemic lupus erythematous. BMJ Case Rep 2016;2016:bcr2016215423. doi:10.1136/bcr-2016-215423. 